\title{
Impact of tissue adhesives on the prevention of anastomotic leakage of colonic anastomoses: an in vivo study
}

\author{
Konstantinos A. Vakalopoulos ${ }^{1,6}$ • Joanna W. A. M. Bosmans ${ }^{2}$. \\ Kevin W. Y. van Barneveld ${ }^{2} \cdot$ Ruben R. M. Vogels $^{2} \cdot$ Geesien S. A. Boersema $^{1}$ • \\ Zhouqiao Wu ${ }^{1,5}$ - Marion J. J. Gijbels ${ }^{3,4}$ - Johannes Jeekel ${ }^{1}$ • Gert-jan Kleinrensink ${ }^{1}$ • \\ Nicole D. Bouvy ${ }^{2}$. Johan F. Lange ${ }^{1}$
}

Accepted: 5 May 2017/Published online: 23 May 2017

(C) The Author(s) 2017. This article is an open access publication

\begin{abstract}
Background Tissue adhesives (TA) may be useful to strengthen colorectal anastomoses, thereby preventing anastomotic leakage (AL). Previous studies have identified cyanoacrylate (CA) TAs as the most promising colonic anastomotic sealants. This study investigates the protective effects of sealing colonic anastomoses with various CAs.

Materials and methods Fifty-five Wistar rats underwent laparotomy and transection of the proximal colon. An anastomosis was created with 4 interrupted sutures followed by either application of Histoacryl Flexible, Omnex, Glubran 2, or no TA seal. An additional control group was included with a 12suture anastomosis and no TA seal. After 7 days, the rats were sacrificed and scored for the presence of $\mathrm{AL}$ as the main outcome. Secondary outcomes were the occurrence of bowel
\end{abstract}

Konstantinos A. Vakalopoulos

k.a.vakalopoulos@gmail.com

1 Department of Surgery, Erasmus University Medical Center Rotterdam, Rotterdam, The Netherlands

2 Department of Surgery, Research Institute NUTRIM, Maastricht University Medical Center, Maastricht, The Netherlands

3 Department of Pathology and Molecular Genetics, Research Institute CARIM, Maastricht University Medical Center, Maastricht, The Netherlands

4 Department of Medical Biochemistry, Academic Medical Center, University of Amsterdam, Amsterdam, The Netherlands

5 Ward I of Gastrointestinal Cancer Center, Key Laboratory of Carcinogenesis and Translational Research (Ministry of Education), Peking University Cancer Hospital \& Institute, Beijing 100142, China

6 Erasmus University Medical Center, Room Ee-173: Laboratory of Experimental Surgery, Postbus 2040, 3000

CA Rotterdam, The Netherlands obstruction, adhesions, and anastomotic bursting pressure. Histological evaluation was performed.

Results The highest AL rate was found in the Glubran 2 group (7/11), followed by the 4-sutures group without TA (5/11), and the Omnex group (5/11). Histoacryl Flexible showed the lowest $\mathrm{AL}$ rate (2/11). In the control group, only one rat showed signs of AL. Histologically, the highest influx of inflammatory cells was found in the 4-suture group without TA and for Omnex and Glubran 2. Histoacryl Flexible caused more mature collagen deposition when compared to the other TA groups.

Conclusions Histoacryl Flexible showed the lowest leakage rate compared to the other TA groups and to the 4-suture control group. Glubran 2 showed the highest AL rate and a high inflammatory response. Histoacryl Flexible was associated with the presence of more mature collagen and seems to promote anastomotic healing.

Keywords Anastomosis · Colorectal surgery $\cdot$ Anastomotic leakage $\cdot$ Tissue adhesives

\section{Introduction}

Recently, the idea of sealing an anastomosis externally with a tissue adhesive is gaining popularity and has been linked to promising results [1]. The benefit of such a technique is that a surgeon can create an anastomosis in a conventional manner using sutures or staples and perform an intra-operative anastomotic patency test, before applying an extra layer of protection on the serosal surface of the anastomosed colon. Of the various available tissue adhesives (TAs), a special interest has arisen for cyanoacrylate (CA) TAs [2]. CA is a type of chemical polymer, also known as "superglue." Several experimental studies have been performed using CA glues to prevent AL, yielding ambiguous results $[1,2]$. This may be partly 
due to a large spectrum of experimental methodology in the various studies, in which large differences exist in the used animal models, TA dosage, and experimental end points. This is a well-recognized problem in the field of experimental research on colorectal anastomoses [3]. In the current study, we use a rat model to simulate high rates of AL based on the creation of a mechanically insufficient colonic anastomosis, sealed by a protective barrier of one of three different CA glues. The aim of this study is to identify promising CAs for the prevention of AL, which may prevent the intraperitoneal leakage of bowel contents.

\section{Methods}

\section{Study design}

Three clinically available CAs were included in this study: Histoacryl Flexible (B. Braun, D; n-butyl-2-CA and a softener), Omnex (Ethicon, USA; 2-octyl/butyl lactoyl CA), Glubran 2 (GEM S.r.l., IT; n-butyl-2 CA and methacryloxy sulfolane), or no TA seal. A positive, 12-suture, control group as well as a negative, 4-suture control group were included, which were not sealed by a TA. Rat allocation was performed in a randomized manner using a lottery system. Follow-up was 7 days.

\section{Animals}

Male specified-pathogen-free Wistar rats (250-350 g) were housed at the Central Animal Facility of the Maastricht University Medical Center, the Netherlands. The experimental protocol complied with the Dutch Animal Experimentation Act and was approved by the local Animal Experimental Committee.

\section{Surgical procedure}

A 5-cm midline incision was made; the cecum was identified and exteriorized onto moist sterile gauzes. The ascending colon was transected $2 \mathrm{~cm}$ distally to the cecum, without damaging the mesenteric vessels. An insufficient end-to-end colocolonic anastomosis was created using four evenly distributed polypropylene 6/0 sutures (Prolene; Ethicon, USA). After construction of the anastomosis, in the TA groups, $0.025 \mathrm{~mL}$ of TA was applied evenly to the anastomotic site using the provided applicators. Care was taken to avoid spillage into the abdomen and, if necessary, a blunt needle was used to accurately guide the TA around the anastomosis. Curing time varied based on the manufacturer's guidelines. In the control group, 12 sutures were used instead of 4, obtaining a sufficient anastomosis.

\section{Outcome measures}

The main outcome of the study was anastomotic leakage (AL), including macroscopic anastomotic dehiscence, fecal peritonitis, or large anastomotic abscesses. Upon sacrifice, signs of leakage or TA-related complications were noted, that is, the presence of intraperitoneal abscesses or fecal matter and mechanical ileus. Abscess formation was scored using the following scoring method: (1) one or several perianastomotic millimetric abscesses, (2) abscess covering up to $1 / 4$ of anastomotic circumference, (3) Large abscess; more than $1 / 4$ of anastomotic circumference, and (4) intraabdominal abscess formation. AL was defined as the presence of fecal peritonitis or an abscess score of $>2[4,5]$. The Zühlke score, which depicts the tenacity of intra-abdominal adhesions, was also determined [6]. Anastomotic bursting pressure testing was carried out during which the maximum bursting pressure was recorded for each rat.

\section{Histological evaluation}

Standard hematoxylin-eosin (H\&E) staining was performed. Specimens were scored based on inflammation, fibroblast activity, collagen deposition, and neoangiogenesis according to the Ehrlich and Hunt numerical scale as modified by Phillips et al., in which 0 : no evidence, 1 : occasional evidence, 2 : light scattering, 3: abundant evidence, and 4: confluent cells or fibers [7].

\section{Evaluation of collagen formation}

Tissue sections were stained for collagen using Picro Sirius red, as previously described [8]. It was chosen not to include collagen staining in the 12-suture control group due to ethical reasons, as these findings are well-known and have been reported in numerous recent studies [9-11]. Collagen percentage of anastomotic tissue was calculated. Maturity level of collagen was estimated by calculating the red (mature fibers, collagen type I) versus green (immature fibers, collagen type III) area ratio using the Qwin morphometry-system (Leica QWin V3.5.1, Leica Microsystems).

\section{Statistics}

One-way ANOVA was used in case of continuous variables, with a Bonferroni post-hoc test. A $\chi^{2}$ test or Fisher's exact was used in case of categorical variables. A $p$ value $\leq 0.05$ was considered statistically significant. All analyses were performed using IBM SPSS Statistics, version 21.0 for Mac (IBM SPSS, USA). 


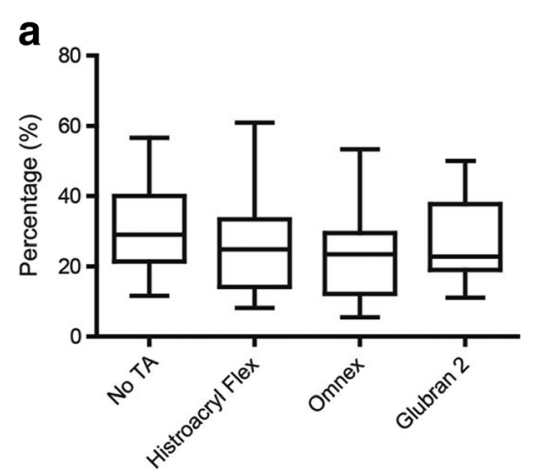

Fig. 1 a Significantly more inflammation occurred in the Omnex and Glubran 2 group compared to the control group. b No differences were found between groups regarding fibroblast acitivity. c More collagen

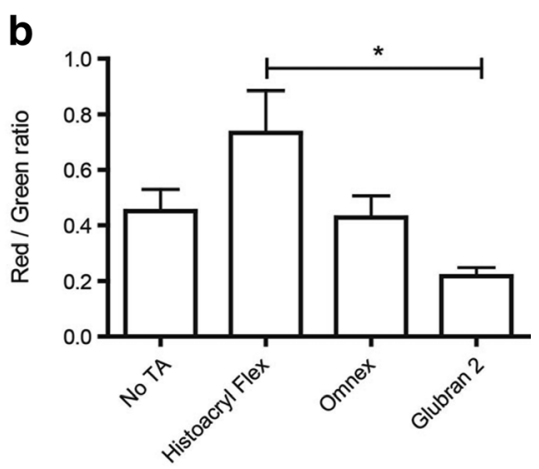

deposition was found in the Histoacryl group and the Omnex group compared to the control group. Neoangiogenesis (d) did not differ between the experimental groups

\section{Results}

\section{Anastomotic leakage}

Both in the 4-suture non-TA group and in the Glubran 2 group, one rat died prior to completion of the follow-up period due to fecal peritonitis caused by AL. Except for these two rats, AL only consisted of the presence of anastomotic abscesses. In the 12-suture control group, AL occurred in one rat, associated with an abscess score of 1 . In the 4-suture non-TA group, four rats showed signs of $\mathrm{AL}$ in the form of abscess formation, with an abscess score of 2 in 3 rats and an abscess score of 4 in 1 rat. In the TA groups, there was a large difference in AL-rate. Glubran 2 had the highest AL rate, consisting of 1 total anastomotic dehiscence and subsequent fecal peritonitis, and 6 cases of abscess formation. Abscess scores in this TA group ranged from 1 to 4.5 rats in the Omnex group and 2 rats in the Histoacryl Flexible group showed signs of AL in the form of abscess formation, with maximum abscess scores of 4 and 2, respectively. Statistical analysis shows that Glubran 2 had a significantly
Fig. 2 a No differences were found between groups in the relative collagen area (quantified as the percentage of total tissue surface). b Maturity of collagen was estimated by calculating the $\mathrm{red} / \mathrm{green}$ ratio, which was significantly higher in the Histoacryl Flexible group compared to the Glubran 2 group, indicating more mature collagen
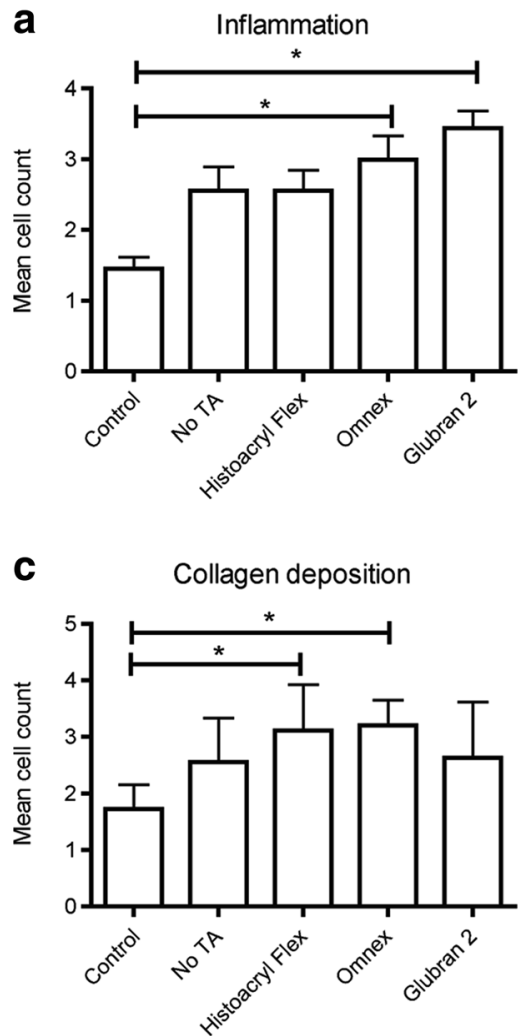
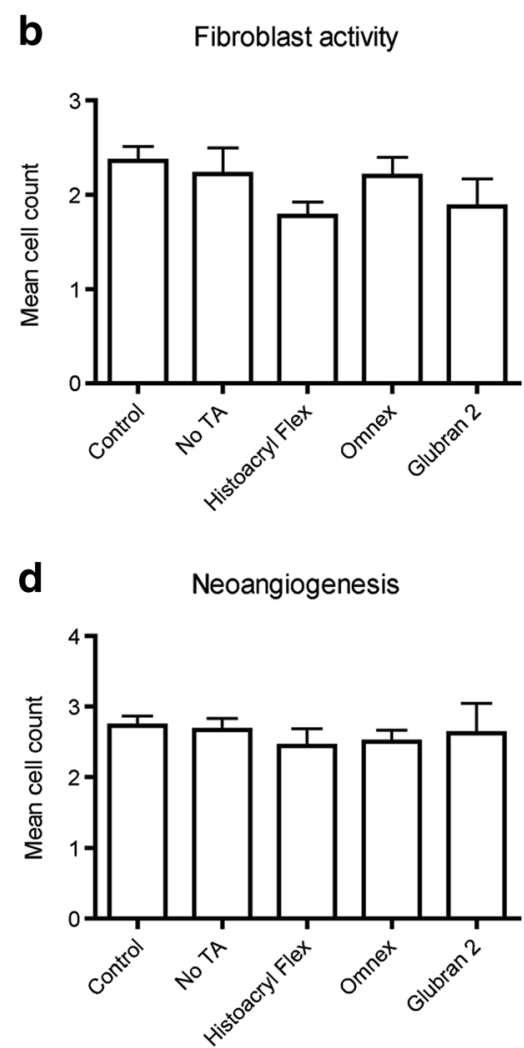
higher amount of abscesses when compared to the positive control group $(p=0.013)$ and Histoacryl Flexible $(p=0.049)$.

\section{Clinical outcomes}

The negative control group, in which no TA was used, showed the highest rate of weight loss. For the TA groups, the Glubran 2 group showed the most weight loss on postoperative day 7 , significantly higher than in the control group $(p<0.01)$ and the Histoacryl Flexible group $(p<0.01)$. Mechanical ileus rate varied significantly between the TA groups, with Glubran 2 showing the highest ileus rate, significantly higher than the negative control group $(p=0.01)$. The number and Zühlke score of adhesions did not differ significantly between the experimental groups.

\section{Anastomotic bursting pressure}

The highest anastomotic bursting pressure (ABP) was found in the 12-suture control group $(272 \mathrm{mmHg} \pm 70)$ and differed significantly from the 4-suture no TA group (147 $\mathrm{mmHg} \pm 37$, $p<0.01)$. The use of TA resulted in an increase in ABP in all 3 TA groups; however, no statistically significant differences were found. The highest increase in ABP was found in the Histoacryl Flexible group $(217 \mathrm{mmHg} \pm 53)$, followed by the Glubran 2 group $(205 \mathrm{mmHg} \pm 67)$. Omnex showed the lowest ABP of the TA groups $(173 \mathrm{mmHg} \pm 69)$.

\section{Histological evaluation}

Histological evaluation is depicted in Fig. 1.

\section{Discussion}

Overall, there were large differences between AL rates of the various groups. AL mostly presented as peri-anastomotic abscess formation. The described abscess score was used to score the severity and amount of abscesses; in this study, an abscess score of $<2$ was not associated with any clinical symptoms, and therefore not considered clinically relevant. Histoacryl Flexible, a combination of n-butyl-2cyanoacrylate and a softener, showed the lowest rate of AL of the TA groups, occurring in two rats. Furthermore, the maximum abscess score in Histoacryl Flexible was lower than in the other TAs and consisted only of punctiform abscesses around the anastomosis, which did not have any clinical consequences. The histological evaluation showed that this TA resulted in the least inflammation and the highest level of collagen formation and healing of the TA groups. Overall, Histoacryl Flexible showed promising results, with an AL rate comparable to the 12-suture control group, with positive clinical outcomes and improved histological assessment. This TA seems to be a safe and effective colonic sealant (Fig. 2).

Glubran 2, based on an n-butyl-2-cyanoacrylate and methacryloxy sulfolane mixture, showed the poorest results in our study. In terms of AL, the use of this TA resulted in one case of premature death due to fecal peritonitis, as well as the highest rates of abscess formation and abscess severity. Furthermore, its use was associated with a higher incidence of mechanical ileus. Rats in this group showed the most weight loss of all study groups. Glubran 2 induced an extended inflammatory response with mild local muscle lysis as deep as the submucosal colonic layer. This finding was also reported in a previous study by Kayaoglu [12]. Omnex, a 2-octylcyanoacrylate/butyl-lactoyl-cyanoacrylate mixture, showed similar results to the negative control group in terms of $\mathrm{AL}$ rate, clinical effects, mechanical strength and histological analysis. The presence of this TA thus did not improve outcomes nor lead to any complications when used on the colon. As the follow-up time in this study was limited to 7 days, we propose a future study to focus on the long-term effects on these TAs on AL rate and tissue healing.

\section{Compliance with ethical standards}

Conflict of interest The authors declare that they have no conflict of interest.

Open Access This article is distributed under the terms of the Creative Commons Attribution 4.0 International License (http:// creativecommons.org/licenses/by/4.0/), which permits unrestricted use, distribution, and reproduction in any medium, provided you give appropriate credit to the original author(s) and the source, provide a link to the Creative Commons license, and indicate if changes were made.

\section{References}

1. Vakalopoulos KA, Daams F, Wu Z et al (2013) Tissue adhesives in gastrointestinal anastomosis: a systematic review. J Surg Res 180(2):290-300

2. Wu Z, Boersema GS, Vakalopoulos KA et al (2014) Critical analysis of cyanoacrylate in intestinal and colorectal anastomosis. J Biomed Mater Res B Appl Biomater 102(3):635-642

3. Bosmans JW, Moossdorff M, Al-Taher M, van Beek L, Derikx JP, Bouvy ND (2016) International consensus statement regarding the use of animal models for research on anastomoses in the lower gastrointestinal tract. Int J Color Dis 31(5):1021-1030

4. Vakalopoulos KA, Wu Z, Kroese LF et al (2017) Clinical, mechanical, and immunohistopathological effects of tissue adhesives on the colon: an in-vivo study. J Biomed Mater Res B Appl Biomater 105(4):846-854

5. Vakalopoulos KA et al (2016) Sutureless closure of colonic defects with tissue adhesives: an in-vivo study in the rat. Am J Surg 213(1): 151-158

6. Zuhlke HV, Lorenz EM, Straub EM, Savvas V (1990) Pathophysiology and classification of adhesions. Langenbecks Arch Chir Suppl II Verh Dtsch Ges Chir:1009-1016 
7. Kanellos D, Blouhos K, Pramateftakis MG et al (2007) Effect of 5fluorouracil plus interferon on the integrity of colonic anastomoses covering with fibrin glue. World J Surg 31(1):186-191

8. Bloemen JG, Schreinemacher MH, de Bruine AP, Buurman WA, Bouvy ND, Dejong CH (2010) Butyrate enemas improve intestinal anastomotic strength in a rat model. Dis Colon rectum 53(7):10691075

9. Hoeppner J, Wassmuth B, Marjanovic G, Timme S, Hopt UT, Keck $\mathrm{T}$ (2010) Anastomotic sealing by extracellular matrices (ECM) improves healing of colonic anastomoses in the critical early phase. $\mathrm{J}$ Gastrointest Surg 14(6):977-986
10. van der Vijver RJ, van Laarhoven CJ, de Man BM, Lomme RM, Hendriks T (2012) The effect of fibrin glue on the early healing phase of intestinal anastomoses in the rat. Int J Color Dis 27(8): 1101-1107

11. Grommes J, Binnebosel M, Klink CD et al (2013) Comparison of intestinal microcirculation and wound healing in a rat model. $\mathrm{J}$ Investig Surg 26(1):46-52

12. Kayaoglu HA, Ersoy OF, Ozkan N, Celik A, Filiz NO (2009) Effect of n-butyl-2-cyanoacrylate on high-risk colonic anastomoses. Kaohsiung J Med Sci 25(4):177-183 